\title{
Sur la réponse transitoire d'une structure en alliage à mémoire de forme soumise à un impact
}

\author{
Manuel Colleta ${ }^{a}$, Morvan Ouisse, Emmanuel Foltete et Christian Lexcellent \\ Institut FEMTO-ST - Département de Mécanique Appliquée, UMR CNRS 6174, 24 rue de l'Épitaphe, 25000 Besançon, France
}

Reçu le 2 octobre 2008, accepté le 3 mars 2009

\begin{abstract}
Résumé - Dans cet article sont présentées des évolutions du modèle RL nécessaires pour la résolution de problèmes de dynamique rapide. Le modèle initial, basé sur le comportement thermomécanique de l'alliage couplé à l'équation de la chaleur, a été implémenté dans le cadre de la dynamique basses fréquences dans des travaux antérieurs. L'utilisation de ce modèle non-linéaire dans le cadre de la dynamique rapide (réponse de la structure à un impact) conduit à des solutions non physiques ou à des divergences des algorithmes d'intégration temporelle. Ces comportements sont principalement dus aux fortes nonlinéarités des équations régissant les cinétiques de transformation entre les deux phases du matériau (austénite/martensite). L'adjonction d'un terme lissant dans les équations différentielles permet de faire converger les algorithmes sans perturber le comportement du modèle initial. Ce terme, d'un point de vue physique, traduit le fait que la transformation de phase ne se fait pas de façon instantanée, mais à une vitesse de propagation donnée. Les résultats issus d'un calcul éléments-finis considérant une structure bidimensionnelle soumise à un impact sont présentés.
\end{abstract}

Mots clés : Alliages à mémoire de forme / dynamique transitoire / éléments-finis / intégration temporelle / dynamique non-linéaire

\begin{abstract}
On the transient impact response of a structure made of shape memory alloy. In this paper, some improvements in the RL model are presented in order to solve transient dynamics problems. The initial model, based on the thermo mechanics behaviour of alloy coupled with the heat equation has been implemented in a finite-element code for low frequency dynamics in previous works. Using this non linear model for transient dynamics (structural response to impact excitation) leads to non-physical solutions or to non convergence of the time integration algorithm. These behaviours are due to strong nonlinearities of equations that govern the transformation kinetics between both phases (austenite/martensite). Adding a relaxation term in differential equations allows the algorithms to converge without disturbing the original system. This term can be associated to a physical effect which is the propagation velocity of the phase transformation, classically considered as instantaneous in static or quasi-static simulations. The results are shown using a $2 \mathrm{D}$ structure excited by an impact force.
\end{abstract}

Key words: Shape memory alloys / transient dynamics / finite-elements / time integration / non-linear dynamics

\section{Introduction}

Les alliages à mémoires de forme $(\mathrm{AMF})$ sont aujourd'hui très étudiés en tant que matériaux multifonctionnels d'une part en tant qu'actionneurs mécaniques mais aussi pour leur pouvoir amortissant.

L'application de ce type de matériaux pour améliorer les caractéristiques vibro-acoustiques de structures

\footnotetext{
a Auteur pour correspondance :

Manuel.collet@univ-fcomte.fr
}

nécessite une modélisation correcte de leur comportement dynamique. La transformation entre la phase mère appelée austénique et la phase produite martensitique induit un comportement non-linéaire pseudo-élastique du matériau. L'énergie dissipée par le cycle d'hystérésis ainsi créé peut alors être optimisée pour conférer au système mécanique étudié de nouvelles propriétés d'amortissement et/ou d'isolation [11, 12]. La modélisation nécessite une approche complète couplant effets thermiques et mécaniques afin de tenir compte correctement des phénomènes physiques mis en jeu lors de sollicitations 


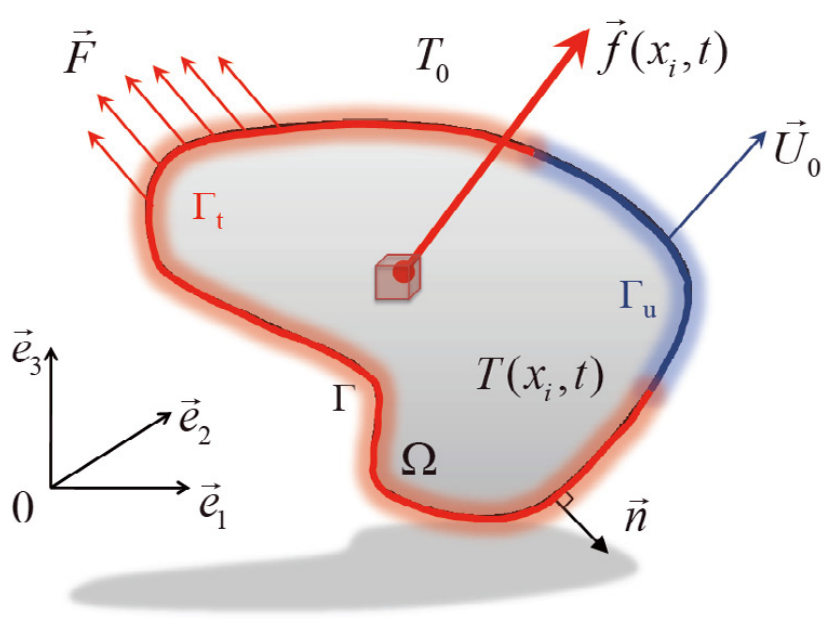

Fig. 1. Domaine et conditions limites.

dynamiques pour lesquels le comportement n'est ni adiabiatique ni isotherme. L'objectif de cet article est alors de présenter une approche de modélisation permettant la simulation de la réponse temporelle transitoire de ces matériaux AMF.

À partir du modèle de Raniecki-Lexcellent, introduit en première partie, nous introduisons dans une seconde partie les éléments de l'implémentation sous COMSOL () d'un modèle complet transitoire thermomécanique. Finalement, nous présentons dans une dernière partie les résultats numériques obtenus pour calculer la réponse au choc d'une poutre AMF en flexion.

\section{Présentation du problème}

Afin de présenter le cadre thermodynamique et constitutif du modèle, on considère une structure en Alliage à Mémoire de Forme (AMF) schématisée par un domaine $\Omega$ de surface fermée $\partial \Omega=\Gamma_{u} \cup \Gamma_{t}$, comme le montre la figure 1 . On note $\vec{n}$ la normale extérieure à cette surface.

La structure de ce domaine peut être chargée mécaniquement par :

- un déplacement imposé $\vec{U}_{0}$ sur le bord $\Gamma_{u}$;

- une densité surfacique $\vec{F}$ sur le bord $\Gamma_{t}$;

- une densité volumique $\vec{f}\left(x_{i}, t\right)$ dans le domaine $\Omega$.

On note $T_{0}$ la température de référence extérieure et $T$ la température au sein du domaine $\Omega$. $\boldsymbol{V}$ est le champ de vitesse d'une particule du domaine $\Omega$.

La résolution d'un tel problème consiste alors à trouver le champ de déplacement $\boldsymbol{u}$, le tenseur des contraintes $\underline{\sigma}$ et la température $T$ satisfaisant les relations suivantes :

Équation de conservation de la masse :

$$
\frac{\mathrm{d} \rho}{\mathrm{d} t}+\rho \operatorname{div} \boldsymbol{V}=0
$$

Équation d'équilibre dynamique :

$$
\rho \frac{\mathrm{d} \boldsymbol{V}}{\mathrm{d} t}=\overrightarrow{\operatorname{div}} \underline{\sigma}+\vec{f}
$$

Équation de conservation de l'énergie :

$$
\rho \frac{\mathrm{d} u}{\mathrm{~d} t}=\underline{\sigma}: \underline{\dot{\varepsilon}}-\operatorname{div} \boldsymbol{q}+r
$$

Conditions limites en effort et en déplacement :

$$
\begin{aligned}
\boldsymbol{u} & =\boldsymbol{U}_{0} \quad \text { sur } \quad \Gamma_{0} \\
\underline{\sigma}(\boldsymbol{n}) & =\boldsymbol{F} \quad \text { sur } \quad \Gamma_{t}
\end{aligned}
$$

Ces équations doivent être complétées par les conditions limites en température (qui seront précisées plus loin), ainsi que par les conditions initiales.

Dans ces équations, $u$ désigne l'énergie interne du système, $\boldsymbol{q}$ le vecteur flux de chaleur, $r$ une source interne de chaleur, $\underline{\varepsilon}$ le tenseur des déformations et $\underline{\sigma}$ le tenseur des contraintes de Cauchy. Ces équations de conservation sont vraies quel que soit le comportement du matériau. Ayant choisi une loi de comportement, on introduit une énergie libre de type Helmholtz contenant l'information sur le comportement du matériau. L'énergie interne $u$ et le tenseur des contraintes de Cauchy $\underline{\sigma}$ sont liés à l'énergie libre de Helmholtz $\phi$ par les relations suivantes :

$$
u=\phi-T \cdot s \quad \sigma=\rho \frac{\partial \phi}{\partial \varepsilon}
$$

où $s$ désigne l'entropie du système. On rappelle alors que dans l'hypothèse des petites perturbations, le tenseur des déformations est lié au champ de déplacement par la relation :

$$
\left.\underline{\varepsilon}=\frac{1}{2}(\underline{\operatorname{grad}} \boldsymbol{u})+\underline{\operatorname{grad}}^{\mathrm{t}} \boldsymbol{u}\right)
$$

Il s'agit alors de définir clairement un potentiel énergie libre de Helmholtz afin de prendre en compte le comportement d'un matériau type AMF lors de la résolution d'un problème de mécanique des structures. L'énergie libre de Helmholtz spécifique aux AMF ainsi que les équations et principes qui en découlent ont été développés dans le cadre de la thermodynamique des processus irréversibles, entre autres par Raniecki et al. [1]. Il s'agit du modèle RL qui est développé ci-après.

\section{Cadre thermodynamique et constitutif du modèle}

\section{1 Énergie libre de Helmholtz}

Afin de définir une fonction énergie libre spécifique de Helmholtz pour les AMF «à l'équilibre contraint », l'énergie libre spécifique d'un mélange de deux phases solides, l'une austénitique (A) et une autre martensitique (M) avec interaction entre phases est utilisée :

$$
\phi=(1-\xi) \phi^{(\mathrm{A})}+\xi \phi^{(\mathrm{M})}+\phi_{\mathrm{int}}
$$

où :

- $\xi$ désigne la fraction volumique de martensitique; 
- $\phi^{(i)}$ désigne l'énergie spécifique d'une phase $i$ austénitique $(i=\mathrm{A})$, ou martensitique $(i=\mathrm{M})$;

- $\phi_{\text {int }}$ désigne l'énergie spécifique d'interaction entre phases.

La fraction volumique de martensite $\xi$ (et donc $(1-\xi)$ celle de l'austénite) est à valeur dans l'intervalle borné $[0,1]$ : pour $\xi=0$, l'alliage est entièrement austénitique et pour $\xi=1$, entièrement martensitique.

L'énergie libre d'interaction entre les deux phases $\phi_{\text {int }}$, par définition, existe si et seulement si il y a cohabitation entre la phase austénitique (A) et la phase martensitique $(\mathrm{M})$, soit $\xi \in] 0,1\left[\right.$. Un choix naturel pour $\phi_{\text {int }}$, introduit par Müller et al. [2,3], consiste à l'annuler pour $\xi=0 \mathrm{ou}$ $\xi=1$, ce qui donne :

$$
\phi_{\text {int }}=\xi(1-\xi) \phi_{\mathrm{it}}(T)
$$

Dans cette continuité, Raniecki et al. [1] ont défini $\phi_{\text {it }}$ comme une énergie d'interaction dépendant de la température en utilisant le concept d'entropie $\bar{s}_{0}$ et d'énergie interne $\bar{u}_{0}$ configurationnelles. Son expression est donnée par la fonction suivante concave dépendante de la température $T$ :

$$
\phi_{\mathrm{it}}(T)=\bar{u}_{0}-T \bar{s}_{0}
$$

Considérons maintenant l'énergie spécifique d'une phase $i$ (austénitique $(i=\mathrm{A})$ ou martensitique $(i=\mathrm{M}))$. L'expression d'une telle énergie spécifique de phase ramenée à un volume élémentaire représentatif (VER) est alors donnée par :

$$
\begin{array}{r}
\phi^{(i)}\left(\underline{\varepsilon}^{(i)}, T\right)=u_{0}^{(i)}-T s_{0}^{(i)}+C_{\mathrm{v}}^{(i)}\left[\left(T-T_{0}\right)-T \ln \left(\frac{T}{T_{0}}\right)\right] \\
+\frac{1}{2 \rho^{(i)}}\left(\underline{\varepsilon}^{(i)}-\underline{\varepsilon}^{\operatorname{tr}(i)}-\underline{\varepsilon}^{\operatorname{th}(i)}\right): \underline{\underline{L}}^{(i)}\left(\underline{\varepsilon}^{(i)}-\underline{\varepsilon}^{\operatorname{tr}(i)}-\underline{\varepsilon}^{\operatorname{th}(i)}\right)
\end{array}
$$

- Le terme $u_{0}^{(i)}-T s_{0}^{(i)}$ constitue la partie « chimique » de l'énergie spécifique de phase où $u_{0}^{(i)}$ et $s_{0}^{(i)}$ sont respectivement l'énergie interne et l'entropie (pour une phase $i$ ) à la température de référence $T_{0}$.

- Le terme $C_{\mathrm{v}}^{(i)}\left[\left(T-T_{0}\right)-T \ln \left(\frac{T}{T_{0}}\right)\right]$ désigne la partie « chaleur spécifique » de l'énergie spécifique de phase où $C_{\mathrm{v}}^{(i)}$ est la chaleur spécifique à volume constant pour une phase $i$.

- Enfin, le terme $\frac{1}{2 \rho^{(i)}}\left(\underline{\varepsilon}^{(i)}-\underline{\varepsilon}^{\operatorname{tr}(i)}-\underline{\varepsilon}^{\operatorname{th}(i)}\right): \underline{\underline{L}}^{(i)}\left(\underline{\varepsilon}^{(i)}-\right.$ $\left.\underline{\varepsilon}^{\operatorname{tr}(i)}-\underline{\varepsilon}^{\operatorname{th}(i)}\right)$ constitue la partie «élastique » de l'énergie libre spécifique de phase où $\rho^{(i)}$ désigne la masse volumique, $\underline{L}^{(i)}$ le tenseur de Hooke du $4^{\mathrm{e}}$ ordre. Chaque terme de déformation est explicité dans la suite, où l'on suppose que les deux phases possèdent des propriétés identiques :

- masse volumique : $\rho^{(\mathrm{A})}=\rho^{(\mathrm{M})}=\rho$;

- caractéristiques élastiques : $\underline{\underline{L}}^{(\mathrm{A})}=\underline{\underline{L}}^{(\mathrm{M})}=\underline{\underline{L}}$;

- coefficient de dilatation thermique $: \bar{\alpha}^{(\mathrm{A})}=\bar{\alpha}^{(\mathrm{M})}=$ $\alpha$;
- chaleur spécifique à volume constant : $C_{\mathrm{v}}^{(\mathrm{A})}=$ $C_{\mathrm{v}}^{(\mathrm{M})}=C_{\mathrm{v}}$.

Le tenseur des déformations élastiques $\underline{\varepsilon}^{\mathrm{el}(i)}$ pour une phase $i$ donnée est défini par :

$$
\underline{\varepsilon}^{\mathrm{el}(i)}=\underline{\varepsilon}^{(i)}-\underline{\varepsilon}^{\operatorname{tr}(i)}-\underline{\varepsilon}^{\operatorname{th}(i)}
$$

Dans cette expression : $\underline{\varepsilon}^{\operatorname{th}(i)}$ représente le tenseur des déformations thermiques issu de la variation de température :

$$
\underline{\varepsilon}^{\mathrm{th}(\mathrm{A})}=\underline{\varepsilon}^{\mathrm{th}(\mathrm{M})}=\underline{\varepsilon}^{\mathrm{th}}=\alpha\left(T-T_{0}\right) \underline{I}
$$

où $\underline{I}$ est le tenseur identité d'ordre 2 .

$\underline{\varepsilon}^{\operatorname{tr}(i)}$ représente la déformation due à la transformation martensitique de l'alliage, qui n'existe donc que pour la phase $i=\mathrm{M}$. L'expression de la déformation pseudoélastique a été proposée par Raniecki et al. [1], qui ont supposé l'existence d'un ensemble de variables internes $h$ représentant les orientations courantes de martensite, dont la transformation se fait sans variation de volume, ce qui permet d'aboutir à :

$$
\underline{\varepsilon}^{\operatorname{tr}(\mathrm{A})}=0, \underline{\varepsilon}^{\operatorname{tr}(\mathrm{M})}=\underline{K}(h), \operatorname{tr}[\underline{K}]=0
$$

où $\operatorname{tr}[\bullet]$ désigne l'opérateur « trace ».

Enfin, le terme $\underline{\varepsilon}^{(i)}$ représente le tenseur des déformations pour la phase $i$.

Sous l'hypothèse d'une loi des mélanges classique, le tenseur des déformations totales peut s'écrire sous la forme suivante :

$$
\underline{\varepsilon}=(1-\xi) \underline{\varepsilon}^{(\mathrm{A})}+\xi \underline{\varepsilon}^{(\mathrm{M})}
$$

Soit, en prenant en compte les expressions précédentes :

$$
\underline{\varepsilon}=\underline{\varepsilon}^{\mathrm{el}(\mathrm{A})}+\underline{\varepsilon}^{\mathrm{th}}+\xi \underline{K}(h)+\xi\left(\underline{\varepsilon}^{\mathrm{el}(\mathrm{M})}-\underline{\varepsilon}^{\mathrm{el}(\mathrm{A})}\right)
$$

D'autre part, le tenseur des contraintes de Cauchy est directement lié au tenseur des déformations élastiques :

$$
\underline{\sigma}^{(i)}=\underline{\underline{L}}^{(i)}\left(\underline{\sigma}^{\mathrm{el}(i)}\right)
$$

Raniecki et al. ont alors formulé l'hypothèse d' «état de contrainte indifférent de l'état de phase » :

$$
\underline{\sigma}^{(i)}=\rho \frac{\partial \phi^{(i)}}{\partial \underline{\varepsilon}^{(i)}} \Rightarrow \underline{\sigma}^{(\mathrm{A})}=\underline{\sigma}^{(\mathrm{M})}=\underline{\sigma}
$$

ce qui implique l'égalité des tenseurs des déformations élastiques des deux phases :

$$
\underline{\varepsilon}^{\mathrm{el}(\mathrm{A})}=\underline{\varepsilon}^{\mathrm{el}(\mathrm{M})}=\underline{\varepsilon}^{\mathrm{el}}
$$

Ainsi, l'expression du tenseur total des déformations se simplifie :

$$
\underline{\varepsilon}=\underline{\varepsilon}^{\mathrm{el}}+\underline{K}(h) \xi+\alpha\left(T-T_{0}\right) \underline{I}
$$

On aboutit finalement à une expression simplifiée du tenseur des déformations pseudo-élastiques, commun aux deux phases :

$$
\underline{\varepsilon}^{\mathrm{tr}}=\underline{K}(h) \xi
$$


C'est donc cette approche qui sera utilisée dans les travaux présentés ici. Notons que d'autres variantes du modèle, ne prenant pas en compte cette hypothèse simplificatrice, ont été développées ces dernières années.

On rappelle finalement que dans le cadre des petites perturbations, le tenseur des déformations est constitué de la partie symétrique du gradient du champ de déplacement, qui sera utilisée dans les premières analyses présentées, permettant de valider l'implémentation numérique du modèle :

$$
\left.\underline{\varepsilon}=\frac{1}{2}(\underline{\operatorname{grad}} \boldsymbol{u})+\underline{\operatorname{grad}}^{\mathrm{t}} \boldsymbol{u}\right)
$$

Tout cela permet d'aboutir à l'expression de l'énergie libre spécifique de Helmholtz pour les AMF :

$$
\begin{aligned}
\phi(\underline{\varepsilon}, T, \xi)= & u_{0}^{(\mathrm{A})}-T s_{0}^{(\mathrm{A})}-\xi \pi_{0}^{\mathrm{f}}(T) \\
& +\frac{1}{2 \rho}\left(\underline{\varepsilon}^{-\underline{\varepsilon}^{\mathrm{tr}}}-\underline{\varepsilon}^{\mathrm{th}}\right): \underline{\underline{L}}\left(\underline{\varepsilon}^{\left.-\underline{\varepsilon}^{\mathrm{tr}}-\underline{\varepsilon}^{\mathrm{th}}\right)}\right. \\
+C_{\mathrm{v}} & {\left[\left(T-T_{0}\right)-T \ln \left(\frac{T}{T_{0}}\right)\right]+\xi(1-\xi) \phi_{\mathrm{it}}(T) }
\end{aligned}
$$

où l'on a introduit la force motrice de transition de phase $\pi_{0}^{\mathrm{f}}(T)$, induite thermiquement à l'état libre de contrainte, qui est définie par les équations suivantes :

$$
\begin{aligned}
\phi^{(\mathrm{A})}-\phi^{(\mathrm{M})} & =\pi_{0}^{\mathrm{f}}(T), \quad \pi_{0}^{\mathrm{f}}(T)=\Delta u_{0}-T \Delta s_{0} \\
\Delta u_{0} & =u_{0}^{(\mathrm{A})}-u_{0}^{(\mathrm{M})}, \quad \Delta s_{0}=s_{0}^{(\mathrm{A})}-s_{0}^{(\mathrm{M})}
\end{aligned}
$$

\subsection{Tenseur associé à la déformation pseudo-élastique}

Dans les équations précédentes, le tenseur $\underline{K}$ n'a pas été explicité. Raniecki et al. [1] ont montré qu'un tenseur dérivant d'un potentiel était une des possibilités d'écriture de ce dernier, et ont proposé une expression correspondant à un choix de comportement symétrique entre la traction et la compression :

$$
\underline{K}=\frac{3}{2} \frac{\operatorname{dev}(\underline{\sigma})}{\underline{\sigma}} \gamma
$$

où $\gamma$ désigne un paramètre matériau représentant la déformation de transformation en traction pure. Dans le cas général, ce paramètre peut avoir deux valeurs différentes en raison de la dissymétrie tractioncompression. Dans le cas où cette dissymétrie peut être négligée, la valeur de $\gamma$ peut être mesurée lors d'un essai de cisaillement pur dans le plan (déformation de cisaillement, contrainte de cisaillement) ou plus simplement, lors d'un essai de traction sur un fil dans le plan (déformation longitudinale, contrainte normale) comme le montre la figure 2.

D'autre part, dans l'expression du tenseur $\underline{K}$ apparaissent l'opérateur déviatorique ainsi que la contrainte équivalente de Von Mises :

$$
\operatorname{dev}(\underline{\sigma})=\underline{\sigma}-\frac{1}{3} \operatorname{tr}(\underline{\sigma}) \underline{I}, \quad \bar{\sigma}=\sqrt{\frac{3}{2} \operatorname{dev}(\underline{\sigma}): \operatorname{dev}(\underline{\sigma})}
$$

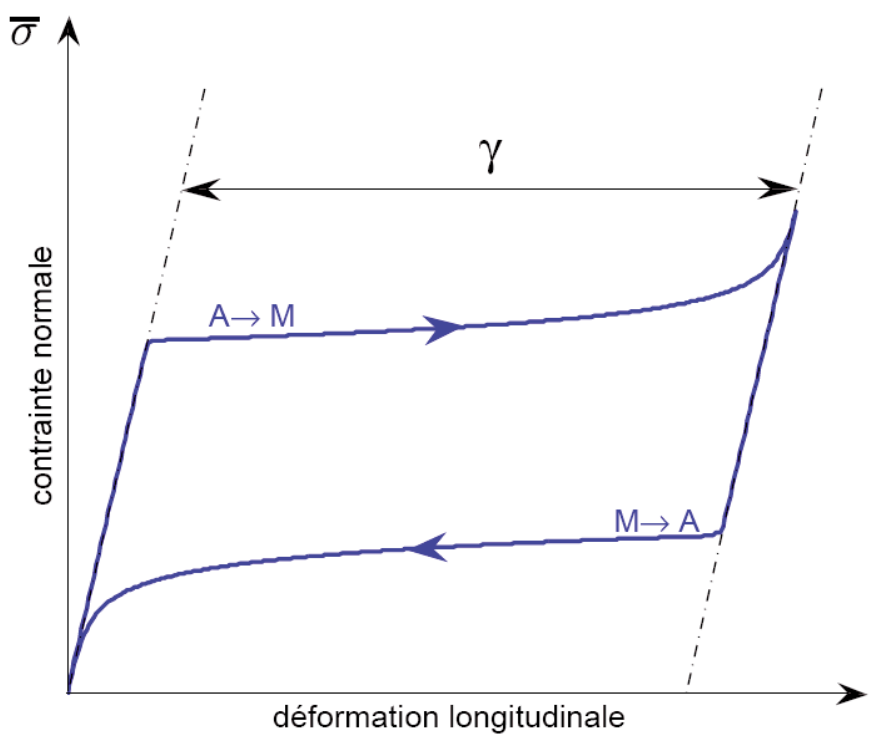

Fig. 2. Identification de la déformation de transformation maximum $\gamma$ à partir d'un essai sur un fil en traction.

De la même façon on peut définir une déformation équivalente au sens de Von Mises :

$$
\underline{\varepsilon}=\sqrt{\frac{2}{3} \operatorname{dev}(\underline{\varepsilon}): \operatorname{dev}(\underline{\varepsilon})}
$$

ce qui permet d'aboutir à l'expression condensée suivante :

$$
\bar{\varepsilon}^{\operatorname{tr}}=\gamma \xi
$$

Cette relation de proportionnalité entre la deformation pseudoélastique équivalente de Von Mises et la fraction volumique de martensite a été proposée par Juhasz et al. [4] et Helm et al. [5]. À l'heure actuelle, des validations expérimentales directes $1 \mathrm{D}$ on été réalisées par différents auteurs [6-8].

Étant données les relations précédentes, la relation entre la déformation et la contrainte équivalente de Von Mises peut finalement s'écrire :

$$
\bar{\sigma}=\frac{3 E}{2(1+\nu)}(\bar{\varepsilon}-\gamma \xi)
$$

\subsection{Processus de transformation et inégalité de Clausius-Duhem}

Afin d'établir les conditions de transformation de phase de l'alliage considéré, il est nécessaire d'écrire le second principe de la thermodynamique, considéré ici sous la forme de l'inégalité de Clausis-Duhem :

$$
\underline{\sigma}: \frac{\mathrm{d} \varepsilon}{\mathrm{d} t}-\rho\left(\frac{\mathrm{d} \phi}{\mathrm{d} t}+s \frac{\mathrm{d} T}{\mathrm{~d} t}\right)-\frac{1}{T} \boldsymbol{q} \cdot \overrightarrow{\operatorname{grad}} T \geq 0
$$

où $s$ désigne l'entropie du système et $\boldsymbol{q}$ le flux de chaleur reçu par le système. L'inégalité de Clausius-Duhem peut être interprétée comme étant une condition nécessaire à 
satisfaire par les différentes variables du système pour que l'évolution du milieu soit physiquement possible ou encore « thermodynamiquement admissible ». En introduisant les concepts de dissipation intrinsèque volumique (notée $D_{\text {int }}$ ) et de dissipation thermique volumique (notée $\left.D_{\text {th }}\right)$, l'inégalité de Clausius-Duhem se réduit à :

$$
D_{\text {int }}+D_{\text {th }} \geq 0
$$

où :

$$
D_{\text {int }}=\underline{\sigma}: \frac{\partial \underline{\varepsilon}}{\partial t}-\rho\left(\frac{\mathrm{d} \phi}{\mathrm{d} t}+s \frac{\mathrm{d} T}{\mathrm{~d} t}\right), D_{\mathrm{th}}=-\frac{1}{T} \boldsymbol{q} \cdot \overrightarrow{\operatorname{grad}} T
$$

Pour toute évolution thermodynamiquement admissible, la dissipation doit être positive ou nulle, ainsi :

$$
D_{\text {int }} \geq 0, \quad D_{\text {th }} \geq 0
$$

En particulier pour la dissipation intrinsèque volumique, on obtient en utilisant les propriétés de différentielle totale de l'énergie libre de Helmholtz :

$D_{\mathrm{int}}=\left(\underline{\sigma}-\rho \frac{\partial \phi}{\partial \underline{\varepsilon}}\right): \underline{\dot{\varepsilon}}-\rho\left[\frac{\partial \phi}{\partial \xi} \dot{\xi}+\left(\frac{\partial \phi}{\partial T}+s\right) \dot{T}\right] \geq 0, \forall \underline{\dot{\varepsilon}}, \dot{T}$

L'expression du tenseur des contraintes de Cauchy a été détaillée plus haut, tandis que l'entropie peut être caractérisée à partir de l'énergie libre de Helmholtz :

$$
\begin{aligned}
s(\underline{\sigma}, T, \xi)=-\frac{\partial \phi}{\partial T}(\underline{\sigma}, T, \xi)= & s_{0}^{(\mathrm{A})}-\xi \Delta s_{0}+C_{\mathrm{v}} \ln \left(\frac{T}{T_{0}}\right) \\
& +\xi(1-\xi) \overline{s_{0}}+\frac{\alpha}{\rho} \underline{\sigma}: \underline{I}
\end{aligned}
$$

Ce qui conduit à une expression condensée de l'inégalité de Clausius-Duhem :

$$
-\rho \frac{\partial \phi}{\partial \xi} \dot{\xi} \geq 0
$$

ou encore :

$$
\pi^{\mathrm{f}} \dot{\xi} \geq 0
$$

Dans cette expression intervient la force thermodynamique associée à la fraction volumique de martensite, que l'on peut exprimer à partir de la contrainte équivalente de Von Mises :

$\pi^{\mathrm{f}}(\underline{\sigma}, T, \xi)=-\frac{\partial \phi}{\partial \xi}(\underline{\varepsilon}, T, \xi)=\frac{\gamma}{\rho} \bar{\sigma}+\pi_{0}^{\mathrm{f}}(T)+\xi(1-2 \xi) \phi_{\mathrm{it}}(T)$

ou de la déformation équivalente :

$\pi^{\mathrm{f}}(\bar{\varepsilon}, T, \xi)=\frac{3 E \gamma}{2 \rho(1+\nu)} \bar{\varepsilon}+\pi_{0}^{\mathrm{f}}(T)-(1-2 \xi) \phi_{\mathrm{it}}-\frac{3 E \gamma^{2}}{2(1+\nu)} \xi$

L'équation (36) permet de définir les conditions de transformation de phase : la force thermodynamique doit être positive ou nulle lors de la transformation directe (A vers $\mathrm{M})$, car il y a production de martensite $(\dot{\xi} \geq 0)$,

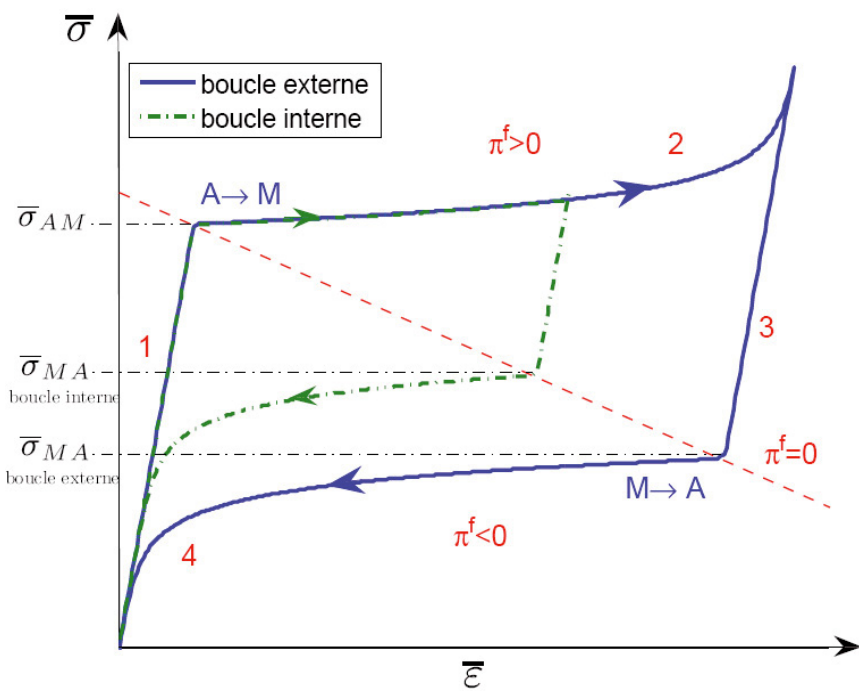

Fig. 3. Représentation schématique du comportement pseudo-élastique des AMF.

et négative ou nulle lors de la transformation inverse (M vers $\mathrm{A})$ où la martensite redevient de l'austénite $(\dot{\xi} \leq 0)$.

Le seuil de tranformation, constituant la limite entre les deux phases, est donc caractérisé par $\pi^{\mathrm{f}}=0$. Le lieu géométrique correspondant aux solutions de cette équation dans le plan des déformations et contraintes équivalentes est une droite dont l'équation est :

$$
\bar{\sigma}=\frac{1}{\frac{4 \phi_{\mathrm{it}}(T)(1+\nu)}{3 E \gamma}-\frac{\gamma}{\rho}}\left(\frac{2 \phi_{\mathrm{it}}(T)}{\gamma} \bar{\varepsilon}+\pi_{0}^{\mathrm{f}}(T)-\phi_{\mathrm{it}}(T)\right)
$$

La représentation graphique de la figure 3 d'un cycle de traction et de l'équation précédente illustre le processus de transformation de l'inégalité de Clausius-Duhem pour une boucle externe et interne. Le tableau suivant récapitule les quatre étapes d'un essai de traction où la transformation est complète.

Dans le cadre d'une transformation complète (où l'austénite se transforme entièrement en martensite en fin de charge, boucle externe $\xi=1$ ) ou partielle (cas où en fin de charge, la transformation de phase est incomplète, boucle interne $\xi \in] 0,1[$ ), la contrainte seuil de début de transformation directe (A vers $\mathrm{M}$ ), notée $\bar{\sigma}_{\mathrm{AM}}$, est définie par :

$$
\pi^{\mathrm{f}}\left(\bar{\sigma}_{\mathrm{AM}}, T, \xi=0\right)=0 \Rightarrow \bar{\sigma}_{\mathrm{AM}}(T)=\frac{\rho}{\gamma}\left(\phi_{\mathrm{it}}(T)-\pi_{0}^{\mathrm{f}}(T)\right)
$$

De même, la contrainte seuil de début de transformation inverse $(\mathrm{M}$ vers $\mathrm{A})$, notée $\bar{\sigma}_{\mathrm{MA}}$ est définie par la relation suivante :

$$
\bar{\sigma}_{\mathrm{MA}}(T)=\frac{\rho}{\gamma}\left((1-2 \xi) \phi_{\mathrm{it}}(T)-\pi_{0}^{\mathrm{f}}(T)\right)
$$

Remarquons que dans le cas d'une boucle externe où la transformation est complète, la contrainte seuil de début de transformation inverse ( $\mathrm{M}$ vers $\mathrm{A})$, on a :

$$
\bar{\sigma}_{\mathrm{MA}}(T)=-\frac{\rho}{\gamma}\left(\phi_{\mathrm{it}}(T)-\pi_{0}^{\mathrm{f}}(T)\right)
$$


Tableau 1. Phase de chargements.

\begin{tabular}{ccccc}
\hline phase & charge/décharge & $\pi^{\mathrm{f}}$ & $\dot{\xi}$ & comportement \\
\hline 1 & charge & négatif & 0 & élastique de l'austénite \\
2 & charge & positif & positif & directe $: A \rightarrow M$ \\
3 & décharge & positif & 0 & élastique de la martensite \\
4 & décharge & négatif & négatif & inverse $: M \rightarrow A$ \\
\hline
\end{tabular}

\subsection{Influence de la température}

La température influence considérablement la transformation martensitique. En effet, la transformation directe (A vers $\mathrm{M}$ ) est exothermique, tandis que la transformation inverse ( $\mathrm{M}$ vers $\mathrm{A}$ ) est endothermique. Expérimentalement, dans [9, 10], Lexcellent et Rejzner ont constaté que le fluage des déformations ou la relaxation des contraintes après un chargement pseudo-élastique s'explique par l'influence de la température sur la transformation martensitique. De même, les travaux de Lim et al. [11] ont montré qu'une augmentation de la vitesse de déformation accroît l'écrouissage du matériau car l'échantillon chauffe davantage pendant l'essai. La variation de la température est régie par l'équation de la chaleur, dans laquelle l'énergie interne peut être exprimée notamment à partir de l'énergie libre de Helmholtz :

$$
u=\phi+T s
$$

En utilisant les relations précédentes, on aboutit à l'équation de la chaleur adaptée au cas de l'alliage AMF :

$C_{\mathrm{v}} \dot{T}-\frac{\lambda}{\rho} \Delta T-\frac{r}{\rho}=\pi^{\mathrm{f}} \dot{\xi}+\dot{\xi} T \Delta s_{0}-\frac{\alpha T}{\rho} \dot{\dot{\sigma}}: \underline{I}-\dot{\xi}(1-2 \xi) \overline{s_{0}}$

où $\lambda$ est le coefficient de conductivité thermique caractérisant la loi de conduction de chaleur isotrope :

$$
\vec{q}=-\lambda \overrightarrow{\operatorname{grad}} T
$$

Pour « fermer » le problème en température, la condition limite associée aux frontières géométriques du domaine est de type convectif :

$$
\frac{\partial T}{\partial n}=-\frac{h_{0}}{\lambda}\left(T-T_{0}\right)
$$

\subsection{Cinétique de transformation}

La dernière étape permettant de fermer le problème est la définition de la cinétique de transformation, qui est caractérisée par les équations donnant les lois d'évolution de la fraction volumique de martensite en fonction des grandeurs thermomécaniques pour les transformations de phase. Par analogie avec la plasticité classique, on postule l'existence de deux fonctions critères $\Psi^{\mathrm{A} \rightarrow \mathrm{M}}$ et $\Psi^{\mathrm{M} \rightarrow \mathrm{A}}$ caractérisant respectivement les transformations directe et inverse. Les processus de transformation ne peuvent alors être effectifs que si les équations de cohérence sont satisfaites, soit :

$$
\begin{aligned}
& \frac{\mathrm{d} \Psi^{\mathrm{A} \rightarrow \mathrm{M}}}{\mathrm{d} t}=0 \text { pour la transformation directe }(\mathrm{A} \rightarrow \mathrm{M}) \\
& \frac{\mathrm{d} \Psi^{\mathrm{M} \rightarrow \mathrm{A}}}{\mathrm{d} t}=0 \text { pour la transformation inverse }(\mathrm{M} \rightarrow \mathrm{A})
\end{aligned}
$$

Il semble naturel de se baser sur la force thermodynamique pour construire ces fonctions critères. On introduit donc deux fonctions $k_{1}$ et $k_{2}$ choisies de manière à retrouver les cinétiques classiques de changement de phases :

$$
\begin{aligned}
& \Psi^{\mathrm{A} \rightarrow \mathrm{M}}(\underline{\sigma}, T, \xi)=\pi^{\mathrm{f}}(\underline{\sigma}, T, \xi)-k_{1}(\xi) \\
& \Psi^{\mathrm{M} \rightarrow \mathrm{A}}(\underline{\sigma}, T, \xi)=-\pi^{\mathrm{f}}(\underline{\sigma}, T, \xi)+k_{2}(\xi)
\end{aligned}
$$

En se basant sur des observations tirées de l'expérience, Raniecki et al. [1] proposent pour les deux fonctions des expressions permettant de garantir les différentes évolutions entre les deux phases :

$$
\begin{aligned}
& k_{1}(\xi)=-\left(A_{1}+B_{1} \xi\right) \ln (1-\xi)+C_{1} \xi \\
& k_{2}(\xi)=\left(A_{2}-B_{2}(1-\xi)\right) \ln (\xi)-C_{2} \xi
\end{aligned}
$$

Parmi les constantes matériau introduites dans ces expressions, seuls les termes $A_{i}$ sont pris en compte, ce qui conduit aux cinétiques de transformation :

$$
\begin{aligned}
\dot{\xi}^{\mathrm{A} \rightarrow \mathrm{M}}(\dot{\bar{\sigma}}, T, \xi) & =\frac{\frac{\gamma \dot{\bar{\sigma}}}{\rho}-\Delta s_{0} \dot{T}}{\frac{A_{1}}{1-\xi}-2 \phi_{\mathrm{it}}} \\
\dot{\xi}^{\mathrm{M} \rightarrow \mathrm{A}}(\dot{\bar{\sigma}}, T, \xi) & =\frac{\frac{\gamma \dot{\bar{\sigma}}}{\rho}-\Delta s_{0} \dot{T}}{\frac{A_{2}}{\xi}-2 \phi_{\mathrm{it}}}
\end{aligned}
$$

Ces cinétiques peuvent également être écrites, de façon équivalente, en fonction de la vitesse de déformation :

$$
\begin{aligned}
\dot{\xi}^{\mathrm{A} \rightarrow \mathrm{M}}(\dot{\bar{\varepsilon}}, T, \xi) & =\frac{3 E \gamma \dot{\bar{\varepsilon}}-2 \rho(1+\nu) \Delta s_{0} \dot{T}}{2 \rho(1+\nu)\left(\frac{A_{1}}{1-\xi}-2 \phi_{\mathrm{it}}\right)+3 E \gamma^{2}} \\
\dot{\xi}^{\mathrm{M} \rightarrow \mathrm{A}}(\dot{\bar{\varepsilon}}, T, \xi) & =\frac{3 E \gamma \dot{\bar{\varepsilon}}-2 \rho(1+\nu) \Delta s_{0} \dot{T}}{2 \rho(1+\nu)\left(\frac{A_{2}}{\xi}-2 \phi_{\mathrm{it}}\right)+3 E \gamma^{2}}
\end{aligned}
$$




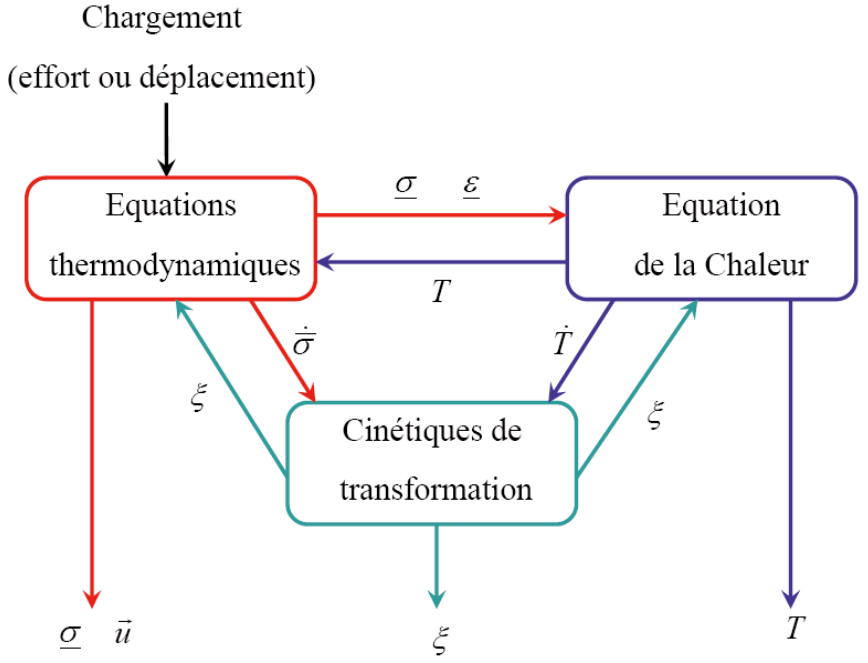

Fig. 4. Couplages entre les différents groupes d'équations.

\section{Implémentation du modèle}

\subsection{Stratégie d'implémentation}

Le modèle qui a été détaillé dans la partie 2 est fortement non-linéaire, et son implémentation dans le cadre de la dynamique rapide n'est disponible dans aucun code de calcul à ce jour. Les travaux effectués ont donc consisté à proposer une implémentation de ce modèle dans le code de calcul COMSOL Multiphysics $^{\mathrm{TM}}$, permettant le couplage entre différentes physiques caractérisées par des équations aux dérivées partielles, dont l'écriture à l'aide d'une formulation variationnelle permet la résolution du problème par la méthode des éléments-finis. Ainsi, sur une même géométrie, trois systèmes d'équations seront résolus de façon couplée, conformément à la figure 4 .

Une des difficultés de l'implémentation réside dans le fait que les différentes équations sont liées les unes aux autres, et que dans le cadre de la résolution de la partie thermomécanique du problème à l'aide d'une formulation en déplacement, il faut être capable d'exprimer la relation de comportement du matériau dans une forme où la contrainte ne dépend que des variables primaires du problème (en l'occurrence, le déplacement de la structure, la température et la fraction volumique de martensite), ce qui n'est pas le cas dans la formulation proposée au-dessus, puisque l'expression de la contrainte fait apparaître, via le terme associé à la transformation de phase, le tenseur $\underline{K}$ qui dépend lui-même de la contrainte :

$$
\underline{\sigma}=\underline{\underline{L}}\left[\underline{\varepsilon}-\frac{3}{2} \frac{\operatorname{dev} \underline{\sigma}}{\bar{\sigma}} \gamma \xi-\alpha\left(T-T_{0}\right) \underline{I}\right]
$$

Différentes techniques existent pour s'affranchir de cette difficulté, l'une d'entre elles consistant à évaluer la contrainte à un instant donné à partir de l'information calculée au pas de temps précédent, ce qui induit un retard, qui, sous certaines hypothèses, peut être négligeable, mais peut évidemment entraîner des erreurs de calcul. La technique qui a été utilisée ici est différente, elle consiste

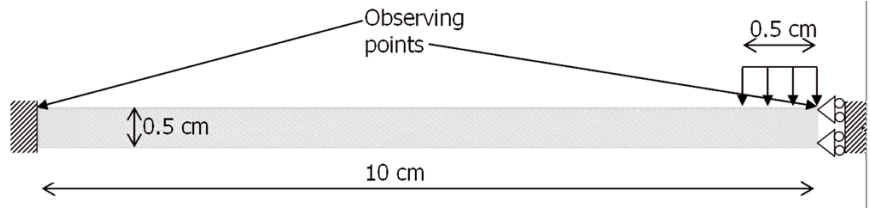

Fig. 5. Géométrie et maillage de la structure utilisée pour le calcul, position des points d'observation.

à remarquer que le rapport entre la partie déviatorique du tenseur des contraintes et la contrainte équivalente de Von Mises peut être relié, dans le cas d'un chargement proportionnel, au déviateur des déformations et à la déformation équivalente au sens de Von Mises :

$$
\underline{K}=\frac{\operatorname{dev} \underline{\varepsilon}}{\bar{\varepsilon}} \gamma=\frac{3}{2} \frac{\operatorname{dev} \underline{\sigma}}{\bar{\sigma}} \gamma
$$

Ainsi, la contrainte a pour expression :

$$
\underline{\sigma}=\underline{\underline{L}}\left[\underline{\varepsilon}-\frac{\operatorname{dev} \underline{\varepsilon}}{\bar{\varepsilon}} \gamma \xi-\alpha\left(T-T_{0}\right) \underline{I}\right]
$$

ce qui permet d'aboutir à une formulation explicite, dans laquelle la contrainte peut être exprimée en fonction des variables du problème. Notons que, dans le cas considéré ici, le chargement envisagé reste proportionnel tant que la géométrie de la zone de chargement ne varie pas et que l'effort appliqué est non nul.

Le matériau qui sera utilisé dans les simulations présentées est un AMF nickel-titane dont les caractéristiques thermomécaniques sont indiquées dans le tableau 2.

\subsection{Validation de l'implémentation dans un cadre quasi-statique isotherme}

Dans le cadre de la thèse de Thiébault $[12,13]$, le modèle décrit dans les pages précédentes a été validé en comparant les résultats de la simulation à des résultats de référence présentés dans [14].

\subsection{Extension du modèle au cas de la dynamique rapide}

L'extension du modèle au cas de la dynamique rapide n'est pas immédiate, car les algorithmes et les formes d'équations utilisées, qui permettent la résolution du problème dans le cas du calcul quasi-statique sont souvent inadaptés aux variations rapides des différents paramètres. Pour procéder à cette extension, nous avons dans un premier temps considéré un modèle isotherme, limité à deux dimensions, sous l'hypothèse des contraintes planes. La structure considérée est illustrée sur la figure 5, il s'agit d'un domaine de forme rectangulaire, en AMF, dont les caractéristiques thermo mécaniques sont données dans le tableau présenté en section 3.1. La structure, de longueur $10 \mathrm{~cm}$ et d'épaisseur $5 \mathrm{~mm}$, est encastrée sur un 
Tableau 2. Paramètres du matériau.

\begin{tabular}{|c|c|c|c|}
\hline Désignation & Notation & Valeur & Unité \\
\hline austenite start & $A_{\mathrm{s}}^{0}$ & 247 & K \\
\hline austenite finish & $A_{\mathrm{f}}^{0}$ & 279 & $\mathrm{~K}$ \\
\hline martensite start & $M_{\mathrm{s}}^{0}$ & 275 & $\mathrm{~K}$ \\
\hline martensite finish & $M_{\mathrm{f}}^{0}$ & 209 & $\mathrm{~K}$ \\
\hline module de Young & $E$ & $52 \times 10^{9}$ & $\mathrm{~Pa}$ \\
\hline coefficient de Poisson & $\nu$ & 0,3 & $\phi$ \\
\hline masse volumique & $\rho$ & 6500 & $\mathrm{~kg} \cdot \mathrm{m}^{-3}$ \\
\hline diff. d'énergie interne & $\Delta u_{0}$ & 8909 & ${\mathrm{~J} . \mathrm{kg}^{-1}}^{-1}$ \\
\hline diff. d'entropie & $\Delta s_{0}$ & 46 & $\mathrm{~J}^{\mathrm{kg}}{ }^{-1} \cdot \mathrm{K}^{-1}$ \\
\hline énergie interne de cohérence & $\bar{u}_{0}$ & 461,5 & ${\mathrm{~J} . \mathrm{kg}^{-1}}^{-1}$ \\
\hline entropie de cohérence & $\bar{s}_{0}$ & 0 & ${\mathrm{~J} . \mathrm{kg}^{-1}}^{-1}$ \\
\hline déformation pseudoélastique maximale & $\gamma$ & $6 \%$ & $\phi$ \\
\hline coefficient de dilatation thermique & $\alpha$ & $11 \times 10^{-6}$ & $\mathrm{~K}^{-1}$ \\
\hline coefficient de conductivité thermique & $\lambda$ & 56,5 & $\mathrm{~W} \cdot \mathrm{m}^{-1} \cdot \mathrm{K}^{-1}$ \\
\hline coefficient de convection & $h$ & 5 & $\mathrm{~W} \cdot \mathrm{m}^{-2} \cdot \mathrm{K}^{-1}$ \\
\hline chaleur spécifique & $C_{\mathrm{v}}$ & 480 & J.K. $\mathrm{kg}^{-1}$ \\
\hline coefficient $A_{1}$ & $A_{1}$ & 699 & J.kg ${ }^{-1}$ \\
\hline coefficient $A_{2}$ & $A_{2}$ & 280 & $\mathrm{J.kg}^{-1}$ \\
\hline
\end{tabular}

bord, guidée sur le côté opposé, et sollicité par un effort (réparti sur une longueur de $5 \mathrm{~mm}$ ) dépendant du temps, correspondant à un effort transitoire « rapide ».

Dans ce modèle, l'amortissement est supposé de type proportionnel $\left(\alpha=\beta=10^{-4}\right)$. Cette modélisation, peu physique, sera améliorée lorsque l'implémentation du modèle AMF sera validée.

Le modèle décrit plus haut permet de modéliser le comportement statique ou dynamique basses fréquences des AMF. Nous avons vu que ce comportement était régi par la force thermodynamique de transformation de phase :

$$
\pi^{\mathrm{f}}(\underline{\sigma}, T, \xi)=\frac{\gamma \sigma_{\mathrm{vm}}}{\rho}+\pi_{0}^{\mathrm{f}}(T)-(1-2 \xi) \phi_{\mathrm{it}}
$$

et que l'on pouvait écrire de façon synthétique la relation de cinétique de transformation :

$$
\dot{\xi}=\mathscr{F}\left(\dot{\sigma}_{\mathrm{vm}}, \pi_{\mathrm{f}}, \xi\right)
$$

La fonction $f$ ainsi définie est nulle sauf dans le cas de la transformation austenite vers martensite $(0<\xi<1$ et $\dot{\sigma}_{\mathrm{vm}}>0$ et $\left.\pi_{\mathrm{f}}(T)>0\right)$ :

$$
\mathscr{F}\left(\dot{\sigma}_{\mathrm{vm}}, \pi_{\mathrm{f}}, \xi\right)=\frac{\gamma \dot{\sigma}_{\mathrm{vm}}}{\rho\left(\frac{A_{1}}{1-\xi}-2 \phi_{\mathrm{it}}\right)}
$$

et lors de la transformation inverse $\left(0<\xi<1, \dot{\sigma}_{\mathrm{vm}} \leq 0\right.$ et $\left.\pi_{\mathrm{f}}(T) \leq 0\right)$ :

$$
\mathscr{F}\left(\dot{\sigma}_{\mathrm{vm}}, \pi_{\mathrm{f}}, \xi\right)=\frac{\gamma \dot{\sigma}_{\mathrm{vm}}}{\rho\left(\frac{A_{2}}{\xi}-2 \phi_{\mathrm{it}}\right)}
$$

Le modèle a donc dans un premier temps été implémenté dans le logiciel COMSOL Multiphysics dans un contexte éléments-finis, de la même façon que celle décrite dans la référence [13], dédiée à la modélisation basses fréquences.
Un schéma d'intégration temporelle implicite a été utilisé pour le solveur temporel, basé sur DASPK [15] (Eulerrétrograde d'ordre variable et de pas variable). Le système d'équations non-linéaires à chaque pas d'intégration est résolu en utilisant un schéma de type Newton-Raphson. Le système linéaire tangent, creux et non symétrique, est finalement résolu en utilisant le solveur direct UMFPACK [16]. Dans un contexte de dynamique rapide, tel que la réponse à un impact, les premiers tests numériques ont montré que la convergence des algorithmes était très difficile à obtenir, et que lorsqu'elle était atteinte, c'était au prix de l'utilisation de nombreux pas temporels et d'un temps de calcul particulièrement long. Une des raisons principales est sans aucun doute la forte nonlinéarité des lois régissant la cinétique de transformation de phase, dont l'expression est régie par les valeurs de la force thermodynamique et de la contrainte équivalente de Von Mises. La stratégie qui a été adoptée pour lisser les difficultés numériques induites par cette loi est d'y ajouter un terme inertiel. D'un point de vue physique, ce terme traduit le fait que la transformation de phase n'est pas instantanée (on trouve dans la littérature un lien entre cette vitesse et la vitesse du son dans l'alliage). Ce terme peut avoir un impact significatif dans le cas où les sollicitations structurales induisent des vitesses proches de la vitesse du son, alors que son effet est négligeable pour les applications quasi-statiques ou dynamiques basses fréquences. La loi régissant la cinétique de transformation de phase a donc été modifiée :

$$
t_{\mathrm{m}}(\xi) \ddot{\xi}+\dot{\xi}=f\left(\sigma_{\mathrm{vm}}, \pi_{\mathrm{f}}, \xi\right)
$$

où le terme supplémentaire $t_{\mathrm{m}}(\xi)$ est une fonction de relaxation qui peut être choisie en fonction de la vitesse du son dans l'alliage. Ce terme agit comme un délai temporal dans la réponse et régularise le comportement de transformation de phase. Dans les tests, $t_{\mathrm{m}}(\xi)$ est une fonction qui est proche de $10^{-3}$ quand le taux de martensite est 


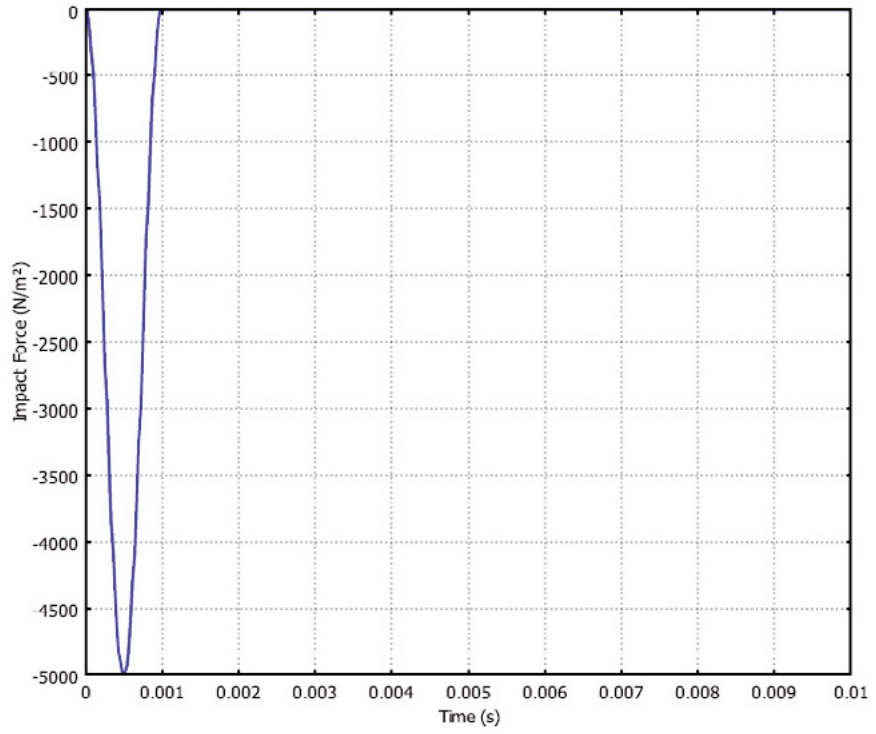

Fig. 6. Évolution temporelle de l'effort d'impact.

supérieur à zéro et inférieur à un, et qui vaut zéro dans tous les autres cas.

L'effort transitoire retenu est présenté sur la figure 6 . Dans ce cas la durée de l'impulsion est de $1 \mathrm{~ms}$.

Le maillage de la structure utilise 2400 éléments triangulaires, la discrétisation étant effectuée par l'intermédiaire de fonctions de formes quadratiques pour le champ de déplacement et de fonctions de formes linéaires pour le champ de fraction volumique de martensite.

Le calcul temporel a été mené sur une durée de $10 \mathrm{~ms}$, le temps de simulation est de l'ordre de $8 \mathrm{~h}$, sur un PC récent $(3,6 \mathrm{GHz}, 4 \mathrm{~Gb}, 64$ bits).

\subsection{1 Évolution temporelle du déplacement}

Le premier résultat présenté illustre l'effet amortissant résultant de la transformation de phase. Sur la figure 7, deux résultats de simulations sont présentés : le premier est relié au calcul incluant le modèle AMF (qui correspond à la modélisation décrite ici), tandis que le second est associé à un modèle linéaire équivalent. Ce dernier est obtenu avec le même modèle dont on force le taux de martensite à zéro. La figure montre la valeur de la flèche au cours du temps, et l'on observe clairement que la raideur apparente du système décroît lorsque la transformation de l'alliage à mémoire de forme est effective. L'amortissement relatif du premier mode est aussi plus important que celui obtenue à l'aide du modèle linéaire.

Un modèle très simple équivalent à 1 DDL peut être utilisé pour quantifier le taux d'amortissement induit par la transformation de phase : le modèle linéaire a une fréquence de $360 \mathrm{~Hz}$ associée à un taux d'amortissement de $11 \%$, tandis que la réponse de l'AMF a une fréquence de $243 \mathrm{~Hz}$ et un taux d'amortissement de $24 \%$. Dans les deux cas, le premier mode est celui qui participe le plus à

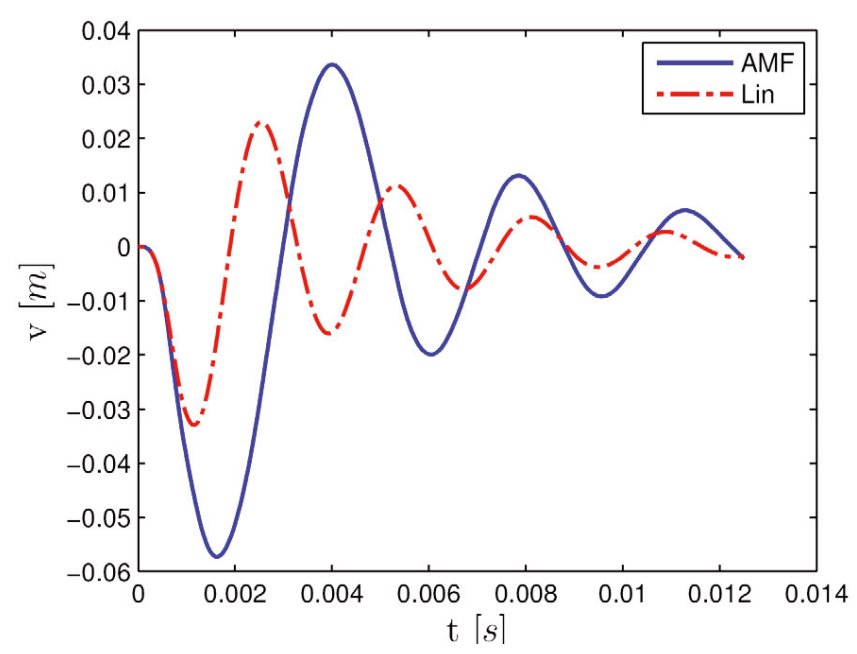

Fig. 7. Évolution temporelle de la flèche maximale pour le calcul AMF/calcul linéaire.

la réponse. Ce système équivalent à un degré de liberté est clairement insuffisant pour une modélisation correcte du système puisqu'il y a une forte non-linéarité de la réponse vis-à-vis de l'amplitude de l'excitation, mais il permet d'obtenir une information qualitative sur l'amortissement apporté par la transformation de phase. Le modèle est donc physiquement cohérent sur ce point.

\subsubsection{Répartition spatiale du taux de martensite}

Sur la figure 8, on peut observer que la réponse est clairement dominée par le premier mode. Cette figure montre le déplacement de la structure et la répartition spatiale de martensite à la fin de l'impact. Le taux de martensite a sa valeur maximale dans les zones où la contrainte est maximale car c'est le premier cycle de l'évolution temporelle, notons cependant que le taux maximum obtenu à cet instant correspond à une zone sur la droite de la structure, alors que la contrainte maximale est atteinte dans la zone de l'encastrement. Nous reviendrons sur ce point plus loin. L'évolution du taux de martensite est gouvernée par la contrainte équivalente de Von Mises et par le signe de la force thermodynamique. On peut observer sur les figures 8 et 9 ce comportement physique : tant que la force thermodynamique est négative, le taux de martensite a une valeur constante nulle. Dès que la force devient positive, le taux augmente, jusqu'à ce que la force devienne négative, car jusqu'à $1 \mathrm{~ms}$, la dérivée temporelle de la contrainte de Von Mises est toujours positive. Ainsi, avant $1 \mathrm{~ms}$, chaque changement de signe de la force thermodynamique induit un point d'inflexion dans l'évolution du taux de martensite.

On peut également observer sur la figure 8 que le modèle est physiquement cohérent, puisque le taux de martensite est supérieur à 1 . 


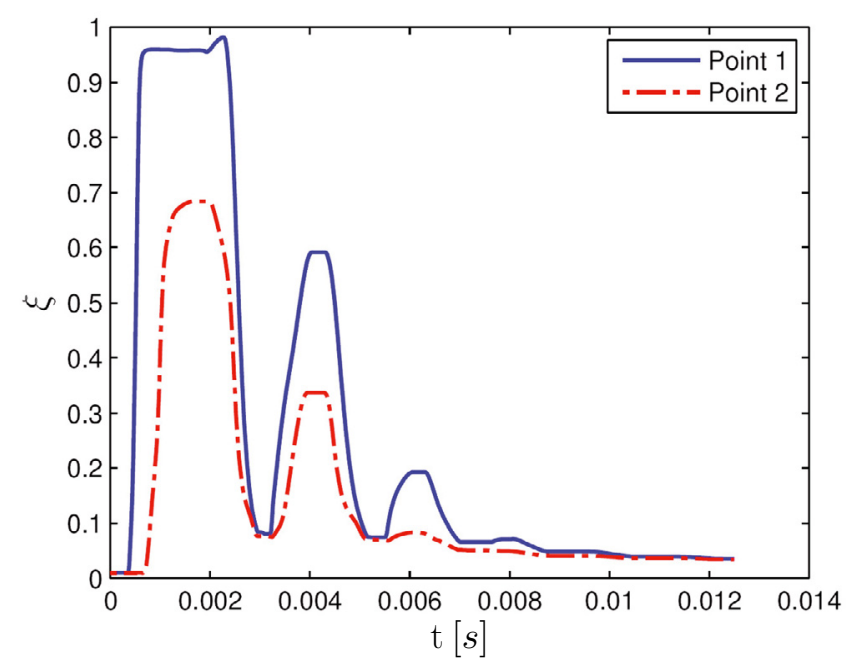

Fig. 8. Évolution temporelle de la répartition de fraction volumique de martensite $\xi$ aux points d'observation.

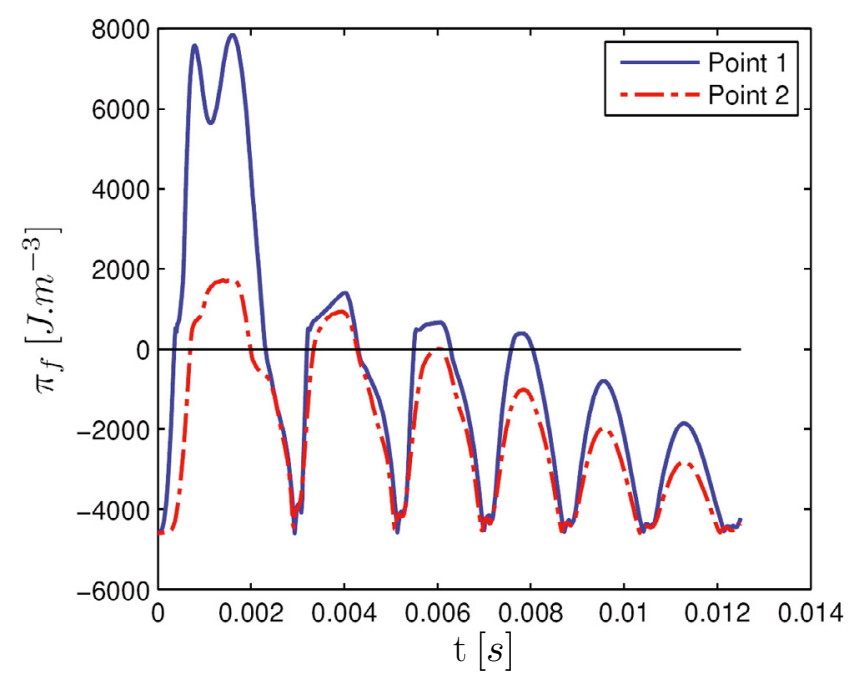

Fig. 9. Évolution temporelle de la force thermodynamique $\pi^{\mathrm{f}}$ aux points d'observation.

\subsection{3 Évolution de la contrainte équivalente de Von Mises}

L'évolution temporelle de la contrainte de Von Mises est d'importance capitale dans le modèle, puisque sa dérivée temporelle gouverne l'évolution du taux de martensite. Une évaluation correcte de cette contrainte est donc nécessaire pour obtenir une évaluation cohérente de l'évolution temporelle du taux de martensite. Sur la figure 10, la répartition spatiale de la contrainte de Von Mises est tracée à $1 \mathrm{~ms}$, et l'on peut observer que sa distribution est physiquement cohérente : la valeur maximale est atteinte sur les faces supérieure et inférieure, au niveau du bord encastré. Dans ce cas particulier, les contraintes ont été correctement évaluées, même pour ces zones particulières grâce au raffinement local du maillage, mais d'autres simulations numériques ont montré une faiblesse du modèle sur ce point, car il est basé sur une formulation en déplacement. Une formulation mixte pourrait

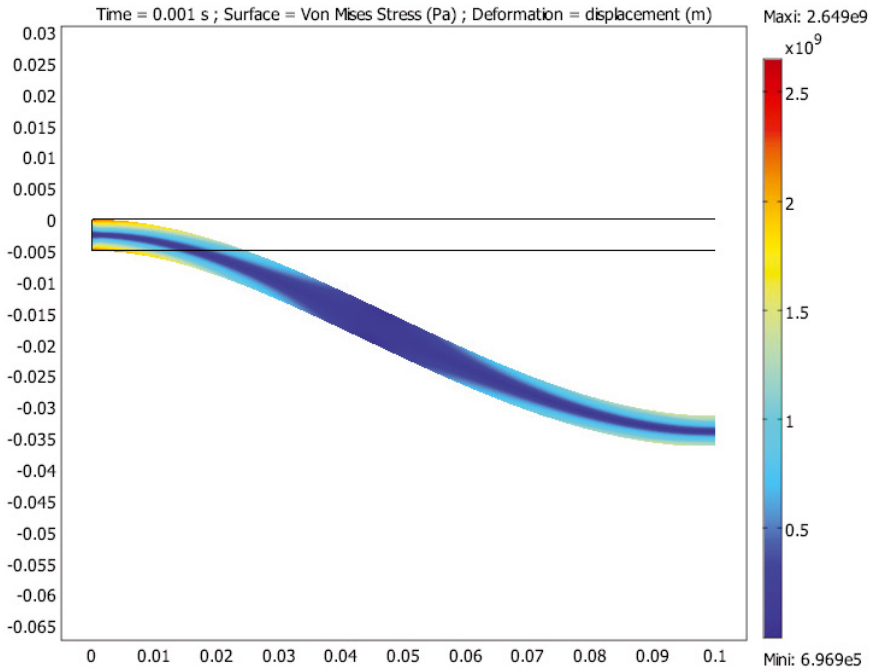

Fig. 10. Déformée et répartition de la contrainte équivalente de Von Mises à $t=1 \mathrm{~ms}$.

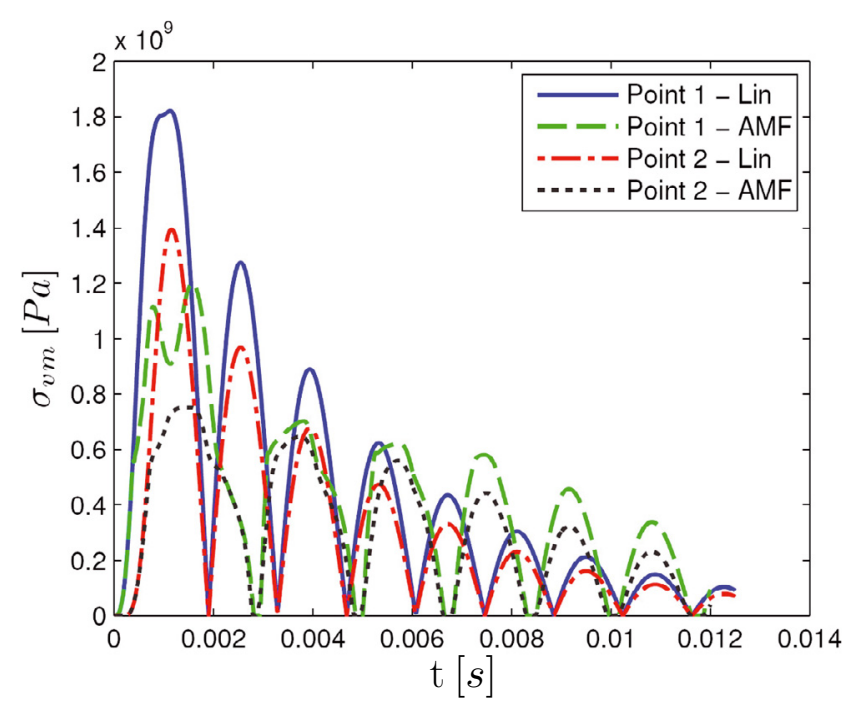

Fig. 11. Évolution temporelle de la contrainte de Von Mises $\bar{\sigma}$ au point d'observation (calcul AMF et calcul linéaire).

être envisagée afin d'obtenir plus efficacement une estimation correcte des contraintes, qui gouvernent la transformation de phase.

La figure 11 présente l'évolution temporelle de la contrainte équivalente de Von Mises sur la partie supérieure de la poutre aux points d'observation 1 et 2 , pour le calcul AMF et pour le modèle linéaire associé. On peut observer que le niveau de contrainte maximal est atteint pour le modèle linéaire, tandis que l'énergie requise pour la transformation de phase induit une valeur plus faible pour la contrainte maximale durant le premier cycle. Les résultats présentés sont cohérents : les évolutions temporelles du système avec et sans transformation présentent le mème décalage fréquentiel que celui observé sur la figure 7 ; le point $1(x=0,1 \mathrm{~cm})$ présente la plus grande valeur de contrainte de Von Mises. 


\section{Conclusion}

L'implémentation du modèle permet donc d'aboutir à des résultats qui rendent compte d'un comportement compatible avec la physique du modèle, que ce soit dans les ordres de grandeur ou les évolutions des différentes grandeurs thermomécaniques. En terme de méthodologie, l'implémentation est donc validée. Il reste à la valider en terme quantitatif par des confrontations avec des mesures in situ. Pour cela il est indispensable de permettre au modèle de capturer les effets de la température, car une transformation isotherme, si elle est envisageable physiquement, ne peut pas rendre compte correctement du comportement du système dans un cas réel, surtout si l'excitation est du type impulsionnel. Avant la validation expérimentale, nous envisageons donc d'étendre le modèle afin de prendre en compte les effets de la température. Dans le même temps, d'autres expressions de la cinétique de transformation de phase seront envisagées, afin d'avoir de meilleurs résultats en terme de convergence des algorithmes d'intégration temporelles, qui, à l'heure d'aujourd'hui et avec le modèle utilisé, ne convergent pas systématiquement.

Remerciements. Ce travail a été en partie financé par l'ADEME via le projet ODIN dans le cadre du programme PREDIT.

\section{Références}

[1] B. Raniecki, C. Lexcellent, K. Tanaka, Thermodynamic models of pseudoelastic behavior of shape memory alloys, Arch. Meh. 44 (1992) 261-284

[2] I. Müller, On the size of the hysteresis in pseudo-elasticity, Continum Mech. Thermodyn. 1 (1989) 125-142

[3] I. Müller, H. Xu, On the pseudoelastic hysteresys, Acta. Met. Mat. 39 (1991) 263-271

[4] L. Juhasz, E. Schnack, O. Hesebeck, H. Andrä, Macroscopic modeling of shape memory alloys under nonproportional thermo-mechanical loadings, J. Intel. Mat. Syst. Struc. 18 (2002) 25-836

[5] D. Helm, P. Haupt, Shape memory behaviour: modelling within continuum thermomechanics, Int. J. Solids Struct. 40 (2003) 827-849
[6] P. Vacher, Étude du comportement pseudoélastique d'alliages à mémoire de forme $\mathrm{CuZnAl}$ polycristallins, Thèse, Université de Franche-Comté, 1991

[7] C. Gonzalez, Étude des comportements électrothermomécaniques et de la stabilisation martensitique d'alliages monocristallins à mémoire de forme base cuivre, Thèse, INSA de Lyon, 2002

[8] T. Kotil, H. Sehitoglu, H. Maier, H. Chumlyakov, Transformation and detwinning induced electrical resistance variations in niticu, Mat. Eng. 329 (2003) 280-289

[9] C. Lexcellent, J. Rejzner, Modeling of the strain rate effect, creep and relaxation of a $\mathrm{Ni}$ - Ti shape memory alloy under tension (compression)-torsional proportional loading in the pseudoelastic range, Smart Mater. Struct. 9 (1998) 613-621

[10] J. Rejzner, Modélisation des alliages à mémoire de forme soumis à des sollicitations multiaxiales ou à des gradients de contrainte, Thèse, Université de Franche-Comté, 2000

[11] T.J. Lim, D.L. Mc Dowell, Mechanical behavior of an NiTi shape memory alloy under axial-torsional proportional and non proportional loading, J. Eng. Mater. Tech. 121 (1999) 9-18

[12] F. Thiebault, De l'étude dynamique des alliages à mémoire de forme à l'optimisation de leur effet amortissant, Thèse, Université de Franche-Comté, 2007

[13] F. Thiebault, C. Lexcellent, M. Collet, E. Foltete, Implementation of a model taking into account the asymmetry between tension and compression, the temperature effects in a finite element code for shape memory alloys structures calculations, Comput. Mat. Sci. 41 (2007) 208221

[14] B. Vieille, De l'élaboration d'un modèle numérique à la prédiction du comportement de structures minces en alliage à mémoire de forme, Thèse, Université de FrancheComté, 2003

[15] P.N. Brown, A.C. Hindmarsh, L.R. Petzold, Using Krylov methods in the solution of large-scale differentialalgebraic systems, SIAM J. Sci. Comput. 15 (1994) 14671488

[16] T.A. Davis, Algorithm 832: UMFPACK V4.3, an unsymmetric-pattern multifrontal method, ACM Trans. Math. Softw. 30 (2004) 196-199 